BULLETIN OF THE POLISH

ACADEMY OF SCIENCES

MATHEMATICS

Vol. 65, No. 2, 2017

\title{
Uniform continuity and normality of metric spaces in $\mathbf{Z F}$
}

by

\author{
Kyriakos KEREMEDIS
}

Presented by Czestaw BESSAGA

Summary. Let $\mathbf{X}=(X, d)$ and $\mathbf{Y}=(Y, \rho)$ be two metric spaces.

(a) We show in $\mathbf{Z F}$ that:

(i) If $\mathbf{X}$ is separable and $f: \mathbf{X} \rightarrow \mathbf{Y}$ is a continuous function then $f$ is uniformly continuous iff for any $A, B \subseteq X$ with $d(A, B)=0, \rho(f(A), f(B))=0$. But it is relatively consistent with $\mathbf{Z F}$ that there exist metric spaces $\mathbf{X}, \mathbf{Y}$ and a continuous, nonuniformly continuous function $f: \mathbf{X} \rightarrow \mathbf{Y}$ such that for any $A, B \subseteq X$ with $d(A, B)=0$, $\rho(f(A), f(B))=0$.

(ii) If $S$ is a dense subset of $\mathbf{X}, \mathbf{Y}$ is Cantor complete and $f: \mathbf{S} \rightarrow \mathbf{Y}$ a uniformly continuous function, then there is a unique uniformly continuous function $F: \mathbf{X} \rightarrow \mathbf{Y}$ extending $f$. But it is relatively consistent with $\mathbf{Z F}$ that there exist a metric space $\mathbf{X}$, a complete metric space $\mathbf{Y}$, a dense subset $S$ of $\mathbf{X}$ and a uniformly continuous function $f: \mathbf{S} \rightarrow \mathbf{Y}$ that does not extend to a uniformly continuous function on $\mathbf{X}$.

(iii) $\mathbf{X}$ is complete iff for any Cauchy sequences $\left(x_{n}\right)_{n \in \mathbb{N}}$ and $\left(y_{n}\right)_{n \in \mathbb{N}}$ in $\mathbf{X}$, if $\overline{\left\{x_{n}: n \in \mathbb{N}\right\}} \cap \overline{\left\{y_{n}: n \in \mathbb{N}\right\}}=\emptyset$ then $d\left(\left\{x_{n}: n \in \mathbb{N}\right\},\left\{y_{n}: n \in \mathbb{N}\right\}\right)>0$.

(b) We show in $\mathbf{Z F}+\mathbf{C A C}$ that if $f: \mathbf{X} \rightarrow \mathbf{Y}$ is a continuous function, then $f$ is uniformly continuous iff for any $A, B \subseteq X$ with $d(A, B)=0, \rho(f(A), f(B))=0$.

1. Notation and terminology. Let $\mathbf{X}=(X, d)$ be a metric space, $x \in X$ and $\varepsilon>0$. As usual, $B(x, \varepsilon)=\{y \in X: d(x, y)<\varepsilon\}$ denotes the open ball in $\mathbf{X}$ with center $x$ and radius $\varepsilon$. Given a non-empty $B \subseteq X$, $\delta(B)=\sup \{d(x, y): x, y \in B\} \in \mathbb{R}_{+} \cup\{\infty\}$ will denote the diameter of $B$.

$\mathbf{X}$ is bounded iff $\delta(X)<\infty$.

Let $\mathcal{U}$ be an open cover of $\mathbf{X}$. We say that $\mathcal{U}$ has a Lebesgue number $\delta>0$ iff for every $A \subseteq X$ with $\delta(A)<\delta$ there exists $U \in \mathcal{U}$ with $A \subseteq U$.

2010 Mathematics Subject Classification: 03E25, 54E35, 54E45, 54E50, 54C20, 54C30.

Key words and phrases: axiom of choice, continuous function, uniformly continuous function, complete, Cantor complete and separable metric spaces.

Received 13 June 2017; revised 26 September 2017.

Published online 23 October 2017. 
$\mathbf{X}$ is Lebesgue (resp. countably Lebesgue) iff every open cover (resp. countable open cover) of $\mathbf{X}$ has a Lebesgue number.

$\mathbf{X}$ is complete iff every Cauchy sequence in $\mathbf{X}$ converges to some element of $X$.

$\mathbf{X}$ is Cantor complete iff $\bigcap\left\{G_{n}: n \in \omega\right\} \neq \emptyset$ for every descending family $\left\{G_{n}: n \in \omega\right\}$ of non-empty closed subsets of $\mathbf{X}$ with $\lim _{n \rightarrow \infty} \delta\left(G_{n}\right)=0$.

$\mathbf{X}$ is $U C$ (resp. strongly $U C$ ) iff every continuous real valued function on $X$ is uniformly continuous (resp. for every metric space $\mathbf{Y}=(Y, \rho)$, every continuous function $f: \mathbf{X} \rightarrow \mathbf{Y}$ is uniformly continuous).

$\mathbf{X}$ is normal iff the distance of any two disjoint, non-empty closed subsets of $\mathbf{X}$ is strictly positive.

$\mathbf{X}$ is sequentially normal (resp. weakly sequentially normal) iff for any two sequences (resp. Cauchy sequences) $\left(x_{n}\right)_{n \in \mathbb{N}}$ and $\left(y_{n}\right)_{n \in \mathbb{N}}$ in $\mathbf{X}$ such that $\left\{x_{n}: n \in \mathbb{N}\right\} \cap \overline{\left\{y_{n}: n \in \mathbb{N}\right\}}=\emptyset$, we have $d\left(\left\{x_{n}: n \in \mathbb{N}\right\},\left\{y_{n}: n \in \mathbb{N}\right\}\right)>0$.

$\mathbf{X}$ is totally bounded iff for every $\varepsilon>0$, there exists an $\varepsilon$-net of $\mathbf{X}$, i.e., a finite subset $\left\{x_{i}: i \leq n\right\}$ of $X$ such that $\bigcup\left\{B\left(x_{i}, \varepsilon\right): i \leq n\right\}=X$.

An infinite set $X$ is Dedekind-infinite, denoted by $\mathbf{D I}(X)$, iff $X$ contains a countably infinite set. Otherwise it is Dedekind-finite. By universal quantifying over $X, \mathbf{D I}(X)$ gives rise to the choice principle IDI: $\forall X(X$ infinite $\rightarrow \mathbf{D I}(X))$, that is, "every infinite set is Dedekind-infinite" [5, Form 9].

Below we list some weak forms of the axiom of choice we shall deal with.

- IDI $(\mathbb{R})$ [5, Form 13]: $\forall X \in \mathcal{P}(\mathbb{R})(X$ infinite $\rightarrow \mathbf{D I}(X))$.

- CAC [5, Form 8]: For every countable family $\mathcal{A}$ of non-empty sets there exists a function $f$ such that $f(x) \in x$ for all $x \in \mathcal{A}$.

- $\mathbf{C A C}_{\text {fin }}$ [5, Form 10]: CAC restricted to countable families of non-empty finite sets. Equivalently (see [5, Form [10 O]], every infinite well ordered family of non-empty finite sets has an infinite subfamily with a choice set.

- $\operatorname{PKW}\left(\aleph_{0}, \geq 2, \infty\right)$ [5, Form 167], Partial Kinna-Wagner principle: Every disjoint family $\mathcal{A}=\left\{A_{i}: i \in \omega\right\}$ with $\left|A_{i}\right| \geq 2$ for all $i \in \omega$ has a partial Kinna-Wagner choice, i.e., there exists an infinite subfamily $\mathcal{B}=\left\{A_{k_{i}}\right.$ : $i \in \omega\}$ of $\mathcal{A}$ and a family $\mathcal{F}=\left\{F_{i}: i \in \omega\right\}$ of non-empty sets such that $F_{i} \subsetneq A_{k_{i}}$ for all $i \in \omega$.

- UCE: For every metric space $\mathbf{X}=(X, d)$ and every complete metric space $\mathbf{Y}=(Y, \rho)$, if $S$ is a dense subset of $\mathbf{X}$ and $f: \mathbf{S} \rightarrow \mathbf{Y}$ is a uniformly continuous function then there exists a unique uniformly continuous extension $F: \mathbf{X} \rightarrow \mathbf{Y}$ of $f$, i.e. $F(s)=f(s)$ for all $s \in S$.

It is known (see e.g. [5]) that in $\mathbf{Z F}$ the $\varepsilon-\delta$ definition of continuity is stronger than the sequential one, and a sequentially closed set may not be closed. We stress that in this paper, continuity will always mean $\varepsilon-\delta$ continuity, and a set is closed iff its complement is open. 
2. Introduction and some preliminary results. Our intended context for reasoning and statements of theorems will be the Zermelo-Fraenkel set theory $\mathbf{Z F}$ without the axiom of choice $\mathbf{A C}$. To stress that a result is proved in $\mathbf{Z F}$ (resp. $\mathbf{Z F}+\mathbf{C A C})$ we shall write $(\mathbf{Z F})(\operatorname{resp} .(\mathbf{Z F}+\mathbf{C A C}))$ at the beginning of the statement.

S. Mrówka [9] has established

Theorem 1. (ZF + CAC) A metric space $\mathbf{X}=(X, \rho)$ is normal iff it is $U C$.

In view of Theorem 1 one may ask whether $\mathbf{C A C}$ is needed for the proof of "normal $\leftrightarrow U C$ ". The following was proved in [6]:

THEOREM $2([6])$.

(a) $(\mathbf{Z F})$ Let $\mathbf{X}=(X, d)$ be a metric space. Each of the following statements implies the one beneath it:

(i) $\mathbf{X}$ is Lebesgue;

(ii) $\mathbf{X}$ is countably Lebesgue;

(iii) $\mathbf{X}$ is $U C$;

(iv) $\mathbf{X}$ is normal.

(b) $(\mathbf{Z F}+\mathbf{C A C})$ (i)-(iv) of (a) are equivalent.

Hence we see that $\mathbf{C A C}$ is not needed for the proof of " $U C \rightarrow$ normal". So, one may ask:

Question 1. Is CAC needed for the proof of "normal $\rightarrow U C$ "?

We show in Theorem 11 that the answer to Question 1 is also negative.

The following well-known characterization of uniform continuity in $\mathbf{Z F}+$ CAC has been established in [2].

Theorem $3([2]) .(\mathbf{Z F}+\mathbf{C A C})$ Let $\mathbf{X}=(X, d)$ and $\mathbf{Y}=(Y, \rho)$ be metric spaces and $f: X \rightarrow Y$ a function. The following are equivalent:

(i) $f$ is uniformly continuous.

(ii) For any subsets $A$ and $B$ of $X$, if $d(A, B)=0$ then $\rho(f(A), f(B))=0$.

(iii) For any countable subsets $A$ and $B$ of $X$, if $d(A, B)=0$ then $\rho(f(A), f(B))=0$.

The next result shows that some parts of the proof of Theorem 3 can be given in $\mathbf{Z F}$.

TheOrem 4. (ZF) Let $\mathbf{X}=(X, d)$ and $\mathbf{Y}=(Y, \rho)$ be metric spaces and $f: \mathbf{X} \rightarrow \mathbf{Y}$ a function.

(i) If for any $A, B \subseteq X, d(A, B)=0$ implies $\rho(f(A), f(B))=0$, then $f$ is continuous. The converse fails. 
(ii) If $f$ is uniformly continuous then for any $A, B \subseteq X, d(A, B)=0$ implies $\rho(f(A), f(B))=0$.

(iii) Assume that every continuous real valued function $g$ on $\mathbf{X}$ satisfies: For any $A, B \subset X, d(A, B)=0$ implies $\rho(g(A), g(B))=0$, where $\rho$ is the usual metric on $\mathbb{R}$. Then $\mathbf{X}$ is sequentially normal.

Proof. The proof of (i) and (ii) is left as an easy exercise for the reader.

(iii) Assume the contrary and fix injective sequences $\left(x_{n}\right)_{n \in \mathbb{N}}$ and $\left(y_{n}\right)_{n \in \mathbb{N}}$ in $\mathbf{X}$ such that $\overline{\left\{x_{n}: n \in \mathbb{N}\right\}} \cap \overline{\left\{y_{n}: n \in \mathbb{N}\right\}}=\emptyset$ and $d\left(\left\{x_{n}: n \in \mathbb{N}\right\},\left\{y_{n}\right.\right.$ : $n \in \mathbb{N}\})=0$. Let $h: \mathbf{Y} \rightarrow \mathbb{R}$, where $Y=A \cup B, A=\overline{\left\{x_{n}: n \in \mathbb{N}\right\}}, B=$ $\overline{\left\{y_{n}: n \in \mathbb{N}\right\}}$, be the function given by

$$
h(x)= \begin{cases}1 & \text { if } x \in A, \\ 0 & \text { if } x \in B .\end{cases}
$$

Clearly, $h$ is continuous. Hence, by the Tietze extension theorem which holds in $\mathbf{Z F}$ (see e.g. [3, Lemma 2]), $h$ extends to a continuous real valued $g$ on $\mathbf{X}$. By our hypothesis, $\rho(g(A), g(B))=0$. However, $\rho(g(A), g(B))=$ $\rho(h(A), h(B))=1$, a contradiction.

The following two theorems characterize, in $\mathbf{Z F}$, the classes of all normal and sequentially normal metric spaces respectively.

Theorem 5. $(\mathbf{Z F})$ Let $\mathbf{X}=(X, \rho)$ be a metric space. Then the following are equivalent:

(i) $\mathbf{X}$ is normal.

(ii) For every metric space $\mathbf{Y}=(Y, d)$, every continuous function $f: \mathbf{X} \rightarrow \mathbf{Y}$, and any $A, B \subseteq X$, if $\rho(A, B)=0$ then $d(f(A), f(B))=0$.

(iii) For every continuous real valued function $f$ on $\mathbf{X}$ and any $A, B \subseteq X$, if $\rho(A, B)=0$ then $d(f(A), f(B))=0$ where $d$ is the usual metric on $\mathbb{R}$.

Proof. (i) $\rightarrow$ (ii) Let $f: \mathbf{X} \rightarrow \mathbf{Y}$ be continuous and fix $A, B \subseteq X$ with $\rho(A, B)=0$. Since $\rho(\bar{A}, \bar{B})=0$, it follows by our hypothesis that $\bar{A} \cap \bar{B}$ $\neq \emptyset$. Hence, $\emptyset \neq f(\bar{A} \cap \bar{B}) \subseteq f(\bar{A}) \cap f(\bar{B})$. By the continuity of $f$ we have $f(\bar{A}) \subseteq \overline{f(A)}$ and $f(\bar{B}) \subseteq \overline{f(B)}$. Hence, $\emptyset \neq \overline{f(A)} \cap \overline{f(B)}$. Therefore, $d(\overline{f(A)}, \overline{f(B)})=0$, and consequently $d(f(A), f(B))=0$ as required.

(ii) $\rightarrow$ (iii) This is straightforward.

(iii) $\rightarrow$ (i) Fix $A, B \subseteq X$ closed and disjoint. We show that $\rho(A, B)>0$. Assume that $\rho(A, B)=0$. It is well known that the function $f: \mathbf{X} \rightarrow \mathbb{R}$ given by

$$
f(x)=\frac{\rho(x, A)}{\rho(x, A)+\rho(x, B)}
$$

is continuous. Hence, by hypothesis, $0=d(f(A), f(B))=d(\{0\},\{1\})=1$, a contradiction. 
Similarly to the proof of Theorem 5 one can establish the following result.

TheOREM 6. $(\mathbf{Z F})$ Let $\mathbf{X}=(X, \rho)$ be a metric space. Then the following are equivalent:

(i) $\mathbf{X}$ is sequentially normal.

(ii) For every metric space $\mathbf{Y}=(Y, d)$, every continuous function $f$ : $\mathbf{X} \rightarrow \mathbf{Y}$, and any countable subsets $A, B \subseteq X$, if $\rho(A, B)=0$ then $d(f(A), f(B))=0$.

(iii) For every continuous real valued function $f$ on $X$, and any countable subsets $A, B \subseteq X$, if $\rho(A, B)=0$ then $d(f(A), f(B))=0$.

Another well-known theorem of $\mathbf{Z F}+\mathbf{C A C}$ regarding uniformly continuous functions states that:

(*) If $\mathbf{X}$ and $\mathbf{Y}$ are metric spaces, $\mathbf{S}$ is a dense subspace of $\mathbf{X}, \mathbf{Y}$ is complete, and $f: \mathbf{S} \rightarrow \mathbf{Y}$ is uniformly continuous, then there exists a unique uniformly continuous extension $F$ of $f$ to all of $\mathbf{X}$.

Let us replace in $(*)$ the word "complete" with "Cantor complete":

(**) If $\mathbf{X}$ and $\mathbf{Y}$ are metric spaces, $\mathbf{S}$ is a dense subspace of $\mathbf{X}, \mathbf{Y}$ is Cantor complete, and $f: \mathbf{S} \rightarrow \mathbf{Y}$ is uniformly continuous, then there exists a unique uniformly continuous extension of $f$ to all of $\mathbf{X}$.

We show in Theorem 15 that $(* *)$ is a theorem of $\mathbf{Z F}$.

The following lemma is stated in [8] without proof and it is attributed to Efremovich. For the reader's convenience, we supply a $\mathbf{Z F}$ proof.

LEMma 7 (Efremovich, [8]). Let $\mathbf{X}=(X, d)$ be a metric space and $\varepsilon>0$. Suppose that $\left(\left(x_{n}, y_{n}\right)\right)_{n \in \mathbb{N}}$ is a sequence in $X \times X$ satisfying $d\left(x_{n}, y_{n}\right) \geq \varepsilon$ for all $n \in \mathbb{N}$. Then there exists a subsequence $\left(\left(x_{k_{n}}, y_{k_{n}}\right)\right)_{n \in \mathbb{N}}$ and $a \kappa \in \mathbb{N}$ such that $d\left(x_{k_{n}}, y_{k_{l}}\right) \geq \varepsilon / \kappa$ for all $n, l \in \mathbb{N}$.

Proof. Let $K=\left\{x_{n}: n \in \mathbb{N}\right\}$ and $F=\left\{y_{n}: n \in \mathbb{N}\right\}$. If $K$ or $F$ is finite then some term of $\left(x_{n}\right)_{n \in \mathbb{N}}$ or $\left(y_{n}\right)_{n \in \mathbb{N}}$ repeats infinitely often. Assume that $\left(x_{k_{n}}\right)_{n \in \mathbb{N}}$ is a constant subsequence of $\left(x_{n}\right)_{n \in \mathbb{N}}$. Clearly, $\left(\left(x_{k_{n}}, y_{k_{n}}\right)\right)_{n \in \mathbb{N}}$ is as required. So, now assume that no term of $\left(x_{n}\right)_{n \in \mathbb{N}}$ or $\left(y_{n}\right)_{n \in \mathbb{N}}$ repeats infinitely often. By passing to subsequences, we may assume that both $\left(x_{n}\right)_{n \in \mathbb{N}}$ and $\left(y_{n}\right)_{n \in \mathbb{N}}$ are injective. We consider the following cases:

(i) Either $K$ or $F$ has an infinite totally bounded subset. Assume that $K$ is totally bounded. In this case we can construct as in [10, Theorem 3.1] a Cauchy subsequence $\left(x_{k_{n}}\right)_{n \in \mathbb{N}}$. Fix $n_{0} \in \mathbb{N}$ such that $d\left(x_{k_{n}}, x_{k_{m}}\right) \leq \varepsilon / 2$ for all $n, m \geq n_{0}$. For all $n, m \geq n_{0}$ we have

$$
\varepsilon \leq d\left(x_{k_{n}}, y_{k_{n}}\right) \leq d\left(x_{k_{n}}, x_{k_{m}}\right)+d\left(x_{k_{m}}, y_{k_{n}}\right) \leq d\left(x_{k_{m}}, y_{k_{n}}\right)+\varepsilon / 2 \text {. }
$$

Therefore, $d\left(x_{k_{m}}, y_{k_{n}}\right) \geq \varepsilon / 2>\varepsilon / 4$. 
(ii) Neither $K$ nor $F$ has an infinite totally bounded subset. Since $K$ is not totally bounded, via an easy induction we construct a subsequence $\left(x_{k_{n}}\right)_{n \in \mathbb{N}}$ such that for all distinct $n, m \in \mathbb{N}, d\left(x_{k_{n}}, x_{k_{m}}\right) \geq \delta_{1}$ for some $\delta_{1}>0$. Since $\left\{y_{k_{n}}: n \in \mathbb{N}\right\}$ is not totally bounded, via a second induction, we construct a subsequence $\left(y_{k_{t_{n}}}\right)_{n \in \mathbb{N}}$ such that $d\left(y_{k_{t_{n}}}, y_{k_{t_{m}}}\right) \geq \delta_{2}$ for some $\delta_{2}>0$ and all distinct $n, m \in \mathbb{N}$. Fix $\kappa \in \mathbb{N} \backslash\{1\}$ such that $\delta=\varepsilon / \kappa<\min \left\{\delta_{1}, \delta_{2}\right\}$. For convenience assume that for all $n \in \mathbb{N}, x_{k_{t_{n}}}=x_{n}$ and $y_{k_{t_{n}}}=y_{n}$. Hence, for all distinct $n, m \in \mathbb{N}$, we have $d\left(x_{n}, x_{m}\right)>\delta, d\left(y_{n}, y_{m}\right)>\delta$ and $d\left(x_{n}, y_{n}\right)>\delta$.

Claim 1. For every $n \in \mathbb{N}$, there is at most one $m \in \mathbb{N}$ such that

$$
\min \left\{d\left(x_{n}, y_{m}\right), d\left(x_{m}, y_{n}\right)\right\}<\delta / 2 .
$$

Proof of Claim 1. Fix $n \in \mathbb{N}$. Assume that there exists $m \in \mathbb{N}$ such that $\min \left\{d\left(x_{n}, y_{m}\right), d\left(x_{m}, y_{n}\right)\right\}<\delta / 2$. We will show that for every $v \in \mathbb{N} \backslash\{n, m\}$, $d\left(x_{n}, y_{v}\right) \geq \delta / 2$ and $d\left(y_{n}, x_{v}\right) \geq \delta / 2$. We consider two cases:

(a) $d\left(x_{n}, y_{m}\right)<\delta / 2$. Since $\delta<d\left(y_{m}, y_{v}\right) \leq d\left(x_{n}, y_{m}\right)+d\left(x_{n}, y_{v}\right)$ and $\delta<d\left(x_{m}, x_{v}\right) \leq d\left(y_{n}, x_{m}\right)+d\left(y_{n}, x_{v}\right)$, it follows that $\delta / 2<\delta-d\left(x_{n}, y_{m}\right) \leq$ $d\left(x_{n}, y_{v}\right)$ and $\delta / 2<\delta-d\left(y_{n}, x_{m}\right) \leq d\left(y_{n}, x_{v}\right)$.

(b) $d\left(x_{m}, y_{n}\right)<\delta / 2$. Working as in (a) we can show that $d\left(x_{n}, y_{v}\right) \geq \delta / 2$ and $d\left(y_{n}, x_{v}\right) \geq \delta / 2$.

Using Claim 1 and a straightforward induction we construct a subsequence $\left(\left(x_{k_{n}}, y_{k_{n}}\right)\right)_{n \in \mathbb{N}}$ such that $d\left(x_{k_{n}}, y_{k_{l}}\right) \geq \delta$ for all $n, l \in \mathbb{N}$, finishing the proof of the lemma.

3. Main results. Our first result in this section shows that the existence of a sequentially normal metric space which is not $U C$ is relatively consistent with ZF.

THEOREM 8. The statement: "Every sequentially normal metric space is normal" implies $\mathbf{I D I}(\mathbb{R})$.

Proof. Aiming for a contradiction, assume that $A$ is a Dedekind-finite subset of $\mathbb{R}$. Let $d$ be the usual metric on $A$ (that is, $d(x, y)=|x-y|$ ). N. Brunner [1] has shown that if there is a Dedekind-finite subset of $\mathbb{R}$ then there also exists a dense one. So, we assume that $\bar{A}=\mathbb{R}$ and $A \cap \mathbb{Q}=\emptyset$. Clearly, any two countable subsets $F, G$ of $A$ with $d(F, G)=0$ intersect. [If $F \cap G=\emptyset$ then $d(F, G)$, being the distance of two finite disjoint sets, is greater than 0.] Hence, $d(f(A), f(B))=0$. So, $\mathbf{A}$ is sequentially normal but not normal (for every $q \in \mathbb{Q}, H=(-\infty, q] \cap A$ and $K=A \cap[q, \infty)$ are disjoint closed subsets of $\mathbf{A}$ such that $d(H, K)=0)$ - a contradiction.

Next we show that CAC implies "every sequentially normal metric space is normal". 
Proposition 9. (CAC) A metric space is sequentially normal iff it is normal.

Proof. Assume $\mathbf{X}=(X, d)$ is a sequentially normal metric space, and fix closed and disjoint subsets $A, B$ of $\mathbf{X}$. Assume for contradiction that $d(A, B)=0$. Clearly, for every $n \in \mathbb{N}$,

$$
W_{n}=\{(x, y) \in A \times B: d(x, y)<1 / n\} \neq \emptyset .
$$

By CAC, for every $n \in \mathbb{N}$, fix $\left(x_{n}, y_{n}\right) \in W_{n}$. Let $H=\left\{x_{n}: n \in \mathbb{N}\right\}$ and $K=\left\{y_{n}: n \in \mathbb{N}\right\}$. Clearly, $\bar{H} \subseteq A, \bar{K} \subseteq B$ and $d(\bar{H}, \bar{K})=0$, contradicting the fact that $\mathbf{X}$ is sequentially normal.

Theorem 10. $(\mathbf{Z F})$ Let $\mathbf{X}=(X, d)$ be a metric space. Then:

(i) If $\mathbf{X}$ is Lebesgue then $\mathbf{X}$ is strongly $U C$.

(ii) $\mathbf{X}$ is weakly sequentially normal iff it is complete.

Proof. (i) See [10, Theorem 7.3]. In the statement of that theorem it is stated that $\mathbf{X}$ is compact but the proof uses only the fact that $\mathbf{X}$ is Lebesgue.

(ii) $(\rightarrow)$ Fix a weakly sequentially normal metric space $\mathbf{X}=(X, d)$. Let $\left(x_{n}\right)_{n \in \mathbb{N}}$ be a Cauchy sequence in $\mathbf{X}$. If $\left(x_{n}\right)_{n \in \mathbb{N}}$ has a limit point then there is nothing to show. So assume that $\left(x_{n}\right)_{n \in \mathbb{N}}$ has no limit points. It follows that any infinite subset of $\left\{x_{n}: n \in \mathbb{N}\right\}$ has no limit points, and consequently it is closed. In particular, $A=\left\{x_{2 n}: n \in \mathbb{N}\right\}$ and $B=\left\{x_{2 n+1}\right.$ : $n \in \mathbb{N}\}$ are closed and $\left(x_{2 n}\right)_{n \in \mathbb{N}},\left(x_{2 n+1}\right)_{n \in \mathbb{N}}$ are Cauchy sequences such that $d(A, B)=0$, contradicting the weak sequential normality of $\mathbf{X}$.

$(\leftarrow)$ Fix Cauchy sequences $\left(x_{n}\right)_{n \in \mathbb{N}}$ and $\left(y_{n}\right)_{n \in \mathbb{N}}$ of $\mathbf{X}$ such that

$$
\overline{\left\{x_{n}: n \in \mathbb{N}\right\}} \cap \overline{\left\{y_{n}: n \in \mathbb{N}\right\}}=\emptyset .
$$

By the completeness of $\mathbf{X}$, let $\lim x_{n}=x \in X$ and $\lim y_{n}=y \in X$. Since $\overline{\left\{x_{n}: n \in \mathbb{N}\right\}}=\{x\} \cup\left\{x_{n}: n \in \mathbb{N}\right\}$ and $\overline{\left\{y_{n}: n \in \mathbb{N}\right\}}=\{y\} \cup\left\{y_{n}: n \in \mathbb{N}\right\}$, it follows that $\overline{\left\{x_{n}: n \in \mathbb{N}\right\}}$ and $\overline{\left\{y_{n}: n \in \mathbb{N}\right\}}$ are compact disjoint subsets of $\mathbf{X}$, and so

$$
d\left(\overline{\left\{x_{n}: n \in \mathbb{N}\right\}}, \overline{\left\{y_{n}: n \in \mathbb{N}\right\}}\right)>0
$$

as required.

REMARK 1. In ZFF, every sequentially normal metric space is weakly sequentially normal, but the converse fails. Indeed, $\mathbb{R}^{2}$ is complete, hence by Theorem 10, weakly sequentially normal. However, it is not sequentially normal: The sets $A=\{(1 / n, n): n \in \mathbb{N}\}$ and $B=\{(0, n): n \in \mathbb{N}\}$ are closed and disjoint, but their distance is 0 .

Next we show that the proof of (ii) $\rightarrow$ (i) of Theorem 3 can be given in $\mathbf{Z F}$ if $\mathbf{Y}$ is separable and in $\mathbf{Z F}+\mathbf{C A C}$ if $\mathbf{Y}$ is not separable. 


\section{THEOREM 11.}

(i) $(\mathbf{Z F})$ Let $\mathbf{X}=(X, d)$ and $\mathbf{Y}=(Y, \rho)$ be metric spaces and $f: \mathbf{X} \rightarrow \mathbf{Y}$ a continuous function. If $\mathbf{Y}$ is separable, then $f$ is uniformly continuous iff for any $A, B \subseteq X$ with $d(A, B)=0, \rho(f(A), f(B))=0$. In particular $\mathbf{X}$ is normal iff it is $U C$.

(ii) $(\mathbf{Z F}+\mathbf{C A C})$ Let $\mathbf{X}=(X, d)$ and $\mathbf{Y}=(Y, \rho)$ be metric spaces and $f: \mathbf{X} \rightarrow \mathbf{Y}$ a continuous function. Then $f$ is uniformly continuous iff for any $A, B \subseteq X$ with $d(A, B)=0, \rho(f(A), f(B))=0$.

Proof. (i) $(\rightarrow)$ This follows from Theorem 4

$(\leftarrow)$ Let $f: \mathbf{X} \rightarrow \mathbf{Y}$ be a continuous function such that for any $A, B \subseteq X$, if $d(A, B)=0$ then $\rho(f(A), f(B))=0$. Aiming for a contradiction, assume that $f$ is not uniformly continuous and fix $\varepsilon>0$ such that for every $n \in \mathbb{N}$, there exist $x_{n}, y_{n} \in X$ such that

$$
d\left(x_{n}, y_{n}\right)<1 / n \text { and } \rho\left(f\left(x_{n}\right), f\left(y_{n}\right)\right) \geq 5 \varepsilon .
$$

Fix a countable dense subset $Q=\left\{q_{n}: n \in \mathbb{N}\right\}$ of $\mathbf{Y}$.

Claim 2. For every $n \in \mathbb{N}$ there exists a pair $\left(p_{n}, q_{n}\right) \in Q^{2}$ with $\rho\left(p_{n}, q_{n}\right)$ $\geq 4 \varepsilon$ and $x, y \in X$ such that

$$
d(x, y)<1 / n, \quad \rho\left(f(x), p_{n}\right)<\varepsilon / 3, \quad \rho\left(f(y), q_{n}\right)<\varepsilon / 3 .
$$

Proof of Claim 2. Fix $n \in \mathbb{N}$. By (1) there exist $x, y \in X$ such that $d(x, y)<1 / n$ and $\rho(f(x), f(y)) \geq 5 \varepsilon$. Fix $p_{n}, q_{n} \in Q$ such that $\rho\left(f(x), p_{n}\right)$ $<\varepsilon / 3$ and $\rho\left(f(y), q_{n}\right)<\varepsilon / 3$. By the triangle inequality,

$$
\begin{aligned}
5 \varepsilon & \leq \rho(f(x), f(y)) \leq \rho\left(f(x), p_{n}\right)+\rho\left(p_{n}, q_{n}\right)+\rho\left(f(y), q_{n}\right) \\
& <\varepsilon / 3+\rho\left(p_{n}, q_{n}\right)+\varepsilon / 3,
\end{aligned}
$$

so $\rho\left(p_{n}, q_{n}\right) \geq 4 \varepsilon$ as required.

For every $n \in \mathbb{N}$ fix a pair $\left(p_{n}, q_{n}\right) \in Q^{2}$ as in Claim 2. By Lemma 7 there exists a subsequence $\left(p_{k_{n}}, q_{k_{n}}\right)_{n \in \mathbb{N}}$ and $v \in \mathbb{N}$ such that $\rho\left(p_{k_{n}}, q_{k_{l}}\right) \geq \varepsilon / v$ for all $n, l \in \mathbb{N}$. For convenience assume that $v=1$. Let

$$
\begin{aligned}
& A=\left\{x \in X: \rho\left(f(x),\left\{p_{k_{n}}: n \in \mathbb{N}\right\}\right)<\varepsilon / 3\right\}, \\
& B=\left\{x \in X: \rho\left(f(x),\left\{q_{k_{n}}: n \in \mathbb{N}\right\}\right)<\varepsilon / 3\right\} .
\end{aligned}
$$

By Claim 2 for every $n \in \mathbb{N}$ there exist $x, y \in X$ such that $d(x, y)<1 / k_{n}$, $\rho\left(f(x), p_{k_{n}}\right)<\varepsilon / 3$, and $\rho\left(f(y), q_{k_{n}}\right)<\varepsilon / 3$. By (2) and (3) we see that $x \in A$ and $y \in B$. Hence, $d(A, B)=0$. However, $\rho(f(A), f(B)) \geq \varepsilon / 3$. Indeed, for every $x \in A$ and $y \in B$ there exist $n, l \in \mathbb{N}$ such that $\rho\left(f(x), p_{k_{n}}\right)<\varepsilon / 3$ and $\rho\left(f(y), q_{k_{l}}\right)<\varepsilon / 3$. Hence,

$$
\begin{aligned}
\varepsilon & \leq \rho\left(q_{k_{l}}, p_{k_{n}}\right) \leq \rho\left(f(x), p_{k_{n}}\right)+\rho(f(x), f(y))+\rho\left(f(y), q_{k_{l}}\right) \\
& <\varepsilon / 3+\rho(f(x), f(y))+\varepsilon / 3 .
\end{aligned}
$$


Therefore, $\rho(f(x), f(y))>\varepsilon / 3$ for all $x \in A$ and $y \in B$, and consequently $\rho(f(A), f(B)) \geq \varepsilon / 3$ as required. This contradicts our assumption.

The second assertion of (i) follows from the first and Theorem 5 .

(ii) It suffices to show $(\leftarrow)$, as the other direction follows from Theorem 4 We shall mimic the proof of (i). Towards a contradiction, fix a continuous, non-uniformly continuous function $f: \mathbf{X} \rightarrow \mathbf{Y}$ such that for any $A, B \subseteq X$, if $d(A, B)=0$ then $\rho(f(A), f(B))=0$. Let $\varepsilon>0$ satisfy: For every $n \in \mathbb{N}$, there are $x, y \in X$ with $d(x, y)<1 / n$ and $\rho(f(x), f(y)) \geq \varepsilon$. Equivalently, if we let $a=f(x)$ and $b=f(y)$,

$$
\forall n \in \mathbb{N}, \exists a, b \in \operatorname{Ran}(f), \quad d\left(f^{-1}(a), f^{-1}(b)\right)<1 / n \text { and } \rho(a, b) \geq \varepsilon .
$$

By CAC, for every $n \in \mathbb{N}$, fix $\left(a_{n}, b_{n}\right) \in \operatorname{Ran}(f)^{2}$ with $d\left(f^{-1}\left(a_{n}\right), f^{-1}\left(b_{n}\right)\right)<$ $1 / n$ and $\rho\left(a_{n}, b_{n}\right) \geq \varepsilon$. By Lemma 7 there exists a subsequence $\left(a_{k_{n}}, b_{k_{n}}\right)_{n \in \mathbb{N}}$ and $v \in \mathbb{N}$ such that $\rho\left(a_{k_{n}}, b_{k_{l}}\right) \geq \varepsilon / v$ for all $n, l \in \mathbb{N}$. Set

$$
A=\bigcup\left\{f^{-1}\left(a_{k_{n}}\right): n \in \mathbb{N}\right\} \quad \text { and } \quad B=\bigcup\left\{f^{-1}\left(b_{k_{n}}\right): n \in \mathbb{N}\right\} .
$$

Clearly, $d(A, B)=0$ but $\rho(f(A), f(B)) \geq \varepsilon / v$, a contradiction.

Corollary 12. $(\mathbf{Z F})$ Let $\mathbf{X}=(X, d)$ be a separable metric space, $\mathbf{Y}=$ $(Y, \rho)$ a metric space and $f: \mathbf{X} \rightarrow \mathbf{Y}$ a function. Then $f$ is uniformly continuous iff for any $A, B \subseteq X$ with $d(A, B)=0, \rho(f(A), f(B))=0$.

Proof. The proof follows at once from Theorem 11 and the fact that the separability of $\mathbf{X}$ implies the separability of $f(\mathbf{X})$.

THEOREM 13.

(i) The statement: "Every normal metric space is strongly UC" implies $\mathbf{P K W}\left(\aleph_{0}, \geq 2, \infty\right)$.

(ii) The statement: "Every countably Lebesgue metric space is strongly UC" implies $\mathbf{P K W}\left(\aleph_{0}, \geq 2, \infty\right)$.

Proof. (i) Assume for contradiction that $\mathbf{P K W}\left(\aleph_{0}, \geq 2, \infty\right)$ fails and fix a disjoint family $\mathcal{A}=\left\{A_{n}: n \in \mathbb{N}\right\}$ of non-empty sets such that no infinite subfamily of $\mathcal{A}$ has a Kinna-Wagner choice set. Let $X=\bigcup\left\{A_{n}: n \in \mathbb{N}\right\}$ and $d: X \times X \rightarrow \mathbb{R}$ be the metric given by

$$
d(x, y)= \begin{cases}0 & \text { if } x=y \\ 1 / n & \text { if } x, y \in A_{n} \\ 1 & \text { if } x \in A_{n}, y \in A_{m} \text { and } n \neq m\end{cases}
$$

Let $\rho$ be the discrete metric on $X$. We claim that $(X, d)$ is normal. It suffices to show that there are no non-empty disjoint subsets $A, B$ of $X$ with $d(A, B)=0$. Assume that $A$ and $B$ are such subsets. Since $d(A, B)=0$, there is an infinite subset $N$ of $\mathbb{N}$ such that for every $n \in N, A \cap A_{n} \neq \emptyset$ 
and $B \cap A_{n} \neq \emptyset$. Hence, $\left\{A \cap A_{n}: n \in N\right\}$ is a partial Kinna-Wagner choice set for $\mathcal{A}$, contradicting our hypothesis. Thus, $(X, d)$ is normal as claimed.

By hypothesis, $(X, d)$ is strongly $U C$. Let $f:(X, d) \rightarrow(X, \rho)$ be given by $f(x)=x, x \in X$. Since $d$ induces the discrete topology on $X$, it follows that $f$ is continuous. Hence, by hypothesis, it is uniformly continuous. Therefore, for $\varepsilon=1$ there is a $k \in \mathbb{N}$ such that for all $x, y \in X$, if $d(x, y)<1 / k$ then $\rho(f(x), f(y))<1$. On the other hand, if $m>k$ and $x, y \in A_{m}, x \neq y$ then $d(x, y)<1 / k$ but $\rho(f(x), f(y)) \geq 1$, a contradiction.

(ii) Let $A, X$ and $d$ be as in the proof of (i). It suffices to show that $\mathbf{X}$ is countably Lebesgue. To see this, fix a countable open cover $\mathcal{U}=\left\{U_{n}: n \in \mathbb{N}\right\}$ of $\mathbf{X}$. Since $\mathcal{A}$ has no Kinna-Wagner choice set, there is $n_{0} \in \mathbb{N}$ such that for all $n \geq n_{0}, A_{n} \subseteq U$ for some $U \in \mathcal{U}$. We claim that $\delta=1 / n_{0}$ is a Lebesgue number of $\mathcal{U}$. Indeed, if $B \subseteq X$ and $\delta(B)<\delta$ then $B$ is a singleton or $B \subseteq A_{k}$ for some $k>n_{0}$. In both cases there is $U \in \mathcal{U}$ with $B \subseteq U$. Hence, $\mathbf{X}$ is countably Lebesgue as required.

Remark 2. The metric space $\mathbf{X}=(X, d)$ in the proof of (i) of Theorem 13 is not Lebesgue. Indeed, the open cover $\mathcal{U}=\{\{x\}: x \in X\}$ of $X$ has no Lebesgue number. [For every $\delta>0$ pick $k \in \mathbb{N}$ with $1 / k<\delta$. Then $\delta\left(A_{k}\right)<\delta$ but $A_{k}$ is included in no member of $\mathcal{U}$.] Thus, in view of the proof of part (ii) of Theorem 13, $\mathbf{X}$ is countably Lebesgue but not Lebesgue.

\section{Theorem 14. CAC implies $\mathbf{U C E}$, and $\mathbf{U C E}$ implies $\mathbf{C A C}_{\text {fin }}$.}

Proof. CAC $\rightarrow$ UCE. The usual proof of AC $\rightarrow$ UCE applies with $\mathbf{C A C}$ in place of $\mathbf{A C}$.

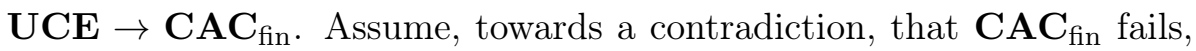
and fix a disjoint family $\mathcal{A}=\left\{A_{n}: n \in \mathbb{N}\right\}$ of non-empty finite sets having no infinite subfamily with a choice function. Let $S=\bigcup\left\{A_{i}: i \in \omega\right\}$ and $X=S \cup\{\infty\}$ where $\infty \notin S$. Let $d: X \times X \rightarrow \mathbb{R}$ be the metric given by

$$
d(x, y)=\left\{\begin{array}{ll}
0 & \text { if } x=y \\
1 / n & \text { if } x \in A_{n}, y \in A_{m} \\
1 / n & \text { if } x \in A_{n}, y=\infty
\end{array} \text { and } n \leq m,\right.
$$

We claim that the subspace $\mathbf{S}$ of $\mathbf{X}$ is complete. To see this, fix a Cauchy sequence $\left(x_{n}\right)_{n \in \mathbb{N}}$ in $\mathbf{S}$. Since $\mathcal{A}$ has no infinite subfamily with a choice set and its members are finite, it follows that $\left\{x_{n}: n \in \mathbb{N}\right\}$ is a finite set. Thus, some term of $\left(x_{n}\right)_{n \in \mathbb{N}}$ repeats infinitely often. Hence, $\left(x_{n}\right)_{n \in \mathbb{N}}$ has a subsequence converging to some $x \in S$. Since $\left(x_{n}\right)_{n \in \mathbb{N}}$ is Cauchy, it converges to $x$, and $\mathbf{S}$ is complete as claimed.

Let $f: \mathbf{S} \rightarrow \mathbf{S}$ be the identity function. Since $f$ is uniformly continuous, by $\mathbf{U C E}$ there exists a uniformly continuous extension $F: \mathbf{X} \rightarrow \mathbf{S}$ of $f$. 
Assume $F(\infty)=x_{\infty} \in A_{k}$. By the uniform continuity of $F$, there exists $\delta>0$ such that for all $x, y \in X$ with $d(x, y)<\delta, d(F(x), F(y))<1 / k$. Fix $m \in \mathbb{N}$ such that $m>k$ and $1 / m<\delta$, and let $y \in A_{m}$. We have $d(y, \infty)=1 / m<\delta$ but $d(F(y), F(\infty))=d\left(y, x_{\infty}\right)=1 / k$, a contradiction.

TheOrem 15. Let $\mathbf{X}=(X, d)$ be a metric space, $S$ a dense subset of $\mathbf{X}$, $\mathbf{Y}=(Y, \rho)$ a Cantor complete metric space and $f: \mathbf{S} \rightarrow \mathbf{Y}$ a uniformly continuous function. Then there exists a unique uniformly continuous function $F: \mathbf{X} \rightarrow \mathbf{Y}$ extending $f$.

Proof. From the uniform continuity of $f$ on $S$ it follows that

$$
\forall n \in \mathbb{N}, \exists \delta_{n}>0, \forall x, y \in S, \quad d(x, y)<\delta_{n} \rightarrow \rho(f(x), f(y))<1 /(6 n) .
$$

Fix a sequence $\left(\delta_{n}\right)_{n \in \mathbb{N}}$ of rationals satisfying (6). For every $x \in X$ let $\mathcal{F}_{x}=\left\{B\left(x, \delta_{n} / 2\right) \cap S: n \in \mathbb{N}\right\}$ and $\mathcal{G}_{x}=\left\{G_{x n}: n \in \mathbb{N}\right\}$ where $G_{x n}=$ $f\left(B\left(x, \delta_{n} / 2\right) \cap S\right)$ for every $n \in \mathbb{N}$. Clearly, $\mathcal{G}_{x}$ is a descending family of subsets of $Y$ such that $\delta\left(G_{x n}\right)<1 / n$ for every $n \in \mathbb{N}$. Hence, by hypothesis, $\bigcap\left\{\overline{G_{x n}}: n \in \mathbb{N}\right\}$ is a singleton of $Y$, say $\left\{\ell_{x}\right\}$. Define $F: \mathbf{X} \rightarrow \mathbf{Y}$ by letting $F(x)=\ell_{x}$ for every $x \in X$.

We claim that $F(s)=f(s)$ for every $s \in S$. Indeed, for every $n \in \mathbb{N}$, we have $s \in B\left(s, \delta_{n} / 2\right) \cap S$. Hence, $f(s) \in G_{s n}$ for all $n \in \mathbb{N}$ and consequently $f(s) \in\left\{\ell_{s}\right\}$, meaning that $f(s)=F(s)$ as required.

We next show that $F$ is uniformly continuous. To see this, fix $\varepsilon>0$ and let $n_{0} \in \mathbb{N}$ satisfy $1 / n_{0}<\varepsilon$. We will show that $\rho(F(x), F(y))<1 / n_{0}$ for any $x, y \in X$ with $d(x, y)<\delta_{n_{0}} / 3$. Fix $x, y \in X$ with $d(x, y)<\delta_{n_{0}} / 3$. We consider the following cases:

(i) $x, y \in S$. In this case the conclusion follows from (6) and the fact that $f(x)=F(x)$ and $f(y)=F(y)$.

(ii) $x \in S, y \notin S$. Fix $x_{1} \in B\left(y, \delta_{n_{0}} / 2\right) \cap S$. Since $f\left(x_{1}\right) \in G_{y n_{0}}$ and $F(y) \in$ $\overline{G_{y n_{0}}}$, it follows that $\rho\left(f\left(x_{1}\right), F(y)\right)<1 /\left(3 n_{0}\right)$. [If $z \in G_{y n_{0}}$ with $z=f(t)$ and $t \in B\left(x, \delta_{n} / 2\right) \cap S$ satisfies $\rho(z, F(y))<1 /\left(6 n_{0}\right)$ then $\rho\left(f\left(x_{1}\right), F(y)\right) \leq$ $\rho\left(f\left(x_{1}\right), z\right)+\rho(z, F(y))<1 /\left(6 n_{0}\right)+1 /\left(6 n_{0}\right)=1 /\left(3 n_{0}\right)$.] We have

$$
d\left(x, x_{1}\right) \leq d(x, y)+d\left(y, x_{1}\right)<\delta_{n_{0}} / 3+\delta_{n_{0}} / 2<\delta_{n_{0}} .
$$

Hence, $\rho\left(f(x), f\left(x_{1}\right)\right)<1 /\left(6 n_{0}\right)$, and consequently

$\rho(f(x), F(y)) \leq \rho\left(f(x), f\left(x_{1}\right)\right)+\rho\left(f\left(x_{1}\right), F(y)\right)<1 /\left(6 n_{0}\right)+1 /\left(3 n_{0}\right)<1 / n_{0}$.

(iii) $x \notin S, y \in S$. This case can be treated as in (ii).

(iv) $x \notin S, y \notin S$. Fix $x_{1} \in B\left(x, \delta_{n_{0}} / 3\right) \cap S$ and $x_{2} \in B\left(y, \delta_{n_{0}} / 3\right) \cap S$. As in case (ii), $\rho\left(f\left(x_{1}\right), F(x)\right)<1 /\left(3 n_{0}\right)$ and $\rho\left(f\left(x_{2}\right), F(y)\right)<1 /\left(3 n_{0}\right)$. Clearly,

$$
d\left(x_{1}, x_{2}\right) \leq d\left(x_{1}, x\right)+d(x, y)+d\left(y, x_{2}\right)<\delta_{n_{0}} / 3+\delta_{n_{0}} / 3+\delta_{n_{0}} / 3=\delta_{n_{0}} .
$$


Hence, by (6), $\rho\left(f\left(x_{1}\right), f\left(x_{2}\right)\right)<1 /\left(6 n_{0}\right)$. We have

$$
\begin{aligned}
\rho(F(x), F(y)) & \leq \rho\left(F(x), f\left(x_{1}\right)\right)+\rho\left(f\left(x_{1}\right), f\left(x_{2}\right)\right)+\rho\left(F(y), f\left(x_{2}\right)\right) \\
& <5 /\left(6 n_{0}\right)<1 / n_{0} .
\end{aligned}
$$

Hence, $F$ is uniformly continuous as claimed.

Finally, we show that $F$ is unique. Assume that $h: \mathbf{X} \rightarrow \mathbf{Y}$ is a uniformly continuous function extending $f$. Suppose there exists $x \in X$ such that $h(x) \neq F(x)$. Then there exists $n \in \mathbb{N}$ such that $h(x) \notin \overline{G_{x n}}$. Hence, there is a $k \in \mathbb{N}$ such that $B(h(x), 1 / k) \cap G_{x n}=\emptyset$. Fix $m>\max \{6 n, k\}$. Since $h$ is continuous and $\bar{S}=X$, there exists $s \in S \cap h^{-1}(B(h(x), 1 / m))$ such that $d(s, x)<\delta_{m} / 2$. Hence, $f(s)=h(s) \in B(h(x), 1 / m)$ and $f(s) \in G_{x m}$, meaning that $B(h(x), 1 / m) \cap G_{x m} \neq \emptyset$. Thus,

$$
\emptyset \neq B(h(x), 1 / m) \cap G_{x m} \subseteq B(h(x), 1 / k) \cap G_{x n}=\emptyset,
$$

a contradiction.

Acknowledgements. I would like to thank the referee for all the useful comments which improved the paper.

\section{References}

[1] N. Brunner, Lindelöf Räume und Auswahlaxiom, Anz. Österreich. Akad. Wiss. Math.Natur. Kl. 119 (1982), 161-165.

[2] R. Cleveland, A global characterization of uniform continuity, Amer. Math. Monthly 80 (1973), 64-66.

[3] P. Howard, K. Keremedis, H. Rubin and J. E. Rubin, Versions of normality and some weak forms of the axiom of choice, Math. Logic Quart. 44 (1998), 367-382.

[4] R. Engelking, General Topology, 2nd ed., Heldermann, Berlin, 1989.

[5] P. Howard and J. E. Rubin, Consequences of the Axiom of Choice, Math. Surveys Monogr. 59, Amer. Math. Soc. Providence, RI, 1998.

[6] K. Keremedis, On metric spaces where continuous real valued functions are uniformly continuous in ZF, Topology Appl. 210 (2016), 366-375.

[7] K. Keremedis and E. Tachtsis, Products of some special compact spaces and restricted forms of AC, J. Symbolic Logic 75 (2010), 996-1006.

[8] S. Kundu and T. Jain, Atsuji spaces: equivalent conditions, Topology Proc. 30 (2006), 301-325.

[9] S. Mrówka, On normal metrics, Amer. Math. Monthly 72 (1965), 998-1001.

[10] J. R. Munkres, Topology, Prentice-Hall, Englewood Cliffs, NJ, 1975.

[11] M. E. Rudin, A new proof that metric spaces are paracompact, Proc. Amer. Math. Soc. 20 (1969), 603.

Kyriakos Keremedis

Department of Mathematics

University of the Aegean

Karlovassi, Samos 83200, Greece

E-mail: kker@aegean.gr 